\title{
DEFINITIONS OF A LINEAR ASSOCIATIVE ALGEBRA BY \\ INDEPENDENT POSTULATES*
}

BY

\author{
LEONARD EUGENE DICKSON
}

\section{Introduction.}

The term linear associative algebra, introduced by Benjamin Peirce, has the same significance as the term system of (higher) complex numbers. $\dagger$ In the usual theory of complex numbers, the coördinates are either real numbers or else ordinary complex quantities. To avoid the resulting double phraseology and to attain an evident generalization of the theory, I shall here consider systems of complex numbers whose coördinates belong to an arbitrary field $F$.

I first give the usual definition by means of a multiplication table for the $n$ units of the system. It employs three postulates, shown to be independent, relating to $n^{3}$ elements of the field $F$.

The second definition is of abstract character. It employs four independent postulates which completely define a system of complex numbers.

The first definition may also be presented in the abstract form used for the second, namely, without the explicit use of units. The second definition may also be presented by means of units. Even aside from the difference in the form of their presentation, the two definitions are essentially different.

\section{First Definition of a System of Complex Numbers.}

Consider $n$ quantities $e_{1}, e_{2}, \cdots, e_{n}$ linearly independent with respect to the field $F$ and having a multiplication-table of the form

$$
e_{i} e_{k s}=\sum_{s=1}^{n} \gamma_{i k s} e_{s} \quad(i, k=1,2, \cdots, n),
$$

where each $\gamma_{i k t}$ belongs to $F$. If $a_{1}, \ldots, a_{n}$ belong to $F$, the expression

$$
a \equiv a_{1} e_{1}+a_{2} e_{2}+\cdots+a_{n} e_{n}
$$

\footnotetext{
* Presented to the Society at the Evanston meeting September 2, 1902. Received for publication September 5, 1902.

† A bibliography of the subject is given by Stupy, Encyklopädie der Mathematischen Wissenschaften, vol. 1, pp. 159-183. Cf. Lie-Scheffers, Continuierliche Gruppen, ch. 21.
} 
is called a complex number with the coördinates $a_{1}, a_{2}, \ldots, a_{n}$. Let

$$
b \equiv b_{1} e_{1}+b_{2} e_{2}+\cdots+b_{n} e_{n}
$$

be a second complex number. Addition and subtraction are defined thus :

$$
a \pm b=\left(a_{1} \pm b_{1}\right) e_{1}+\cdots+\left(a_{n} \pm b_{n}\right) e_{n} .
$$

In accordance with the distributive law, multiplication is defined thus :

It follows from (1) that

$$
a b=\sum_{i, k}^{1, \ldots n} a_{i} b_{k} e_{i} e_{k} .
$$

$$
a b=\sum_{s=1}^{n} u_{s} e_{s}, \quad u_{s} \equiv \sum_{i, k}^{1, \ldots, n} \gamma_{i k s} a_{i} b_{k} .
$$

Whatever be the $n^{3}$ marks $\gamma_{i k s}$ of $F$, we have defined unambiguously certain operations called addition, subtraction and multiplication, which, when applied to any complex numbers with coördinates in $F$, lead uniquely to complex numbers with coördinates in $F$. It remains to impose certain conditions on the $\gamma_{i k s}$ such that there will result a system of complex numbers, viz., one for which the associative law for multiplication holds and for which division (as defined below) may in general be performed uniquely.

In view of (2), the associative law holds always if, and only if,

$$
\left(e_{i} e_{k}\right) e_{l}=e_{i}\left(e_{k} e_{l}\right) \quad(i, k, l=1,2, \cdots, n) .
$$

In view of $(1)$ and the linear independence of $e_{1}, \cdots, e_{n}$, these relations give

$$
\sum_{s=1}^{n} \gamma_{i k s} \gamma_{s l t}=\sum_{s=1}^{n} \gamma_{k l s} \gamma_{i s t} . \quad(i, k, l, t=1, \cdots, n) \text {. }
$$

In order that, for a general complex number $a$ and an arbitrary complex number $b$, it shall be possible to determine uniquely a complex number $x$ such that $a x=b$, the condition is that

$$
\Delta_{a} \equiv\left|\sum_{i=1}^{n} \gamma_{i k s} a_{i}\right| \text { shall not vanish for every } a_{1}, \ldots, a_{n} .
$$

The proof follows from formulæ analogous to (3).

Likewise, in order that it shall be possible to determine uniquely a complex number $y$ such that $y a=b$, the condition is that

$$
\Delta_{a}^{\prime} \equiv\left|\sum_{k=1}^{n} \gamma_{i k s} a_{k}\right| \text { shall not vanish for every } a_{1}, \ldots, a_{n} .
$$


Every system of complex numbers with respect to a field $F$ defines $n^{3}$ marks $\gamma_{i k s}$ of $F$ satisfying the conditions (5), (6), (7). Inversely, such a set of marks $\gamma_{i k s}$ defines a system of complex numbers with respect to $F$.

\section{Independence of the Conditions (5), (6), (7).}

Following the customary method, we exhibit for $j=5,6,7$, a set $S_{j}$ of $n^{3}$ marks $\gamma_{i k s}$ of $F$ for which the $j$ th condition fails while the remaining two conditions are satisfied. It suffices to take $n=2$, whence

$$
\begin{aligned}
& \Delta_{a} \equiv\left|\begin{array}{cc}
\gamma_{111} a_{1}+\gamma_{211} a_{2} & \gamma_{112} a_{1}+\gamma_{212} a_{2} \\
\gamma_{121} a_{1}+\gamma_{221} a_{2} & \gamma_{122} a_{1}+\gamma_{222} a_{2}
\end{array}\right|, \Delta_{a}^{\prime} \equiv\left|\begin{array}{cc}
\gamma_{111} a_{1}+\gamma_{121} a_{2} & \gamma_{112} a_{1}+\gamma_{122} a_{2} \\
\gamma_{211} a_{1}+\gamma_{221} a_{2} & \gamma_{212} a_{1}+\gamma_{222} a_{2}
\end{array}\right| . \\
& S_{5^{\circ}} \quad\left\{\begin{array}{llll}
\gamma_{111}=1, & \gamma_{112}=0, & \gamma_{121}=0, & \gamma_{122}=1, \\
\gamma_{211}=1, & \gamma_{212}=0, & \gamma_{221}=1, & \gamma_{222}=0 . *
\end{array}\right.
\end{aligned}
$$

Then (5) fails for $i=2, k=1, l=2, t=1$, since

$$
\sum_{s=1,2} \gamma_{21 s} \gamma_{s 21}=0, \quad \sum_{s=1,2} \gamma_{12 s} \gamma_{2 s 1}=1
$$

But (6) and (7) hold, since

$$
\begin{aligned}
& \Delta_{a} \equiv\left|\begin{array}{cc}
a_{1}+a_{2} & 0 \\
a_{2} & a_{1}
\end{array}\right|=a_{1}\left(a_{1}+a_{2}\right), \quad \Delta_{a}^{\prime} \equiv\left|\begin{array}{cc}
a_{1} & a_{2} \\
a_{1}+a_{2} & 0
\end{array}\right|=-a_{2}\left(a_{1}+a_{2}\right) . \\
& S_{6} . \quad\left\{\begin{array}{llll}
\gamma_{111}=1, & \gamma_{112}=0, & \gamma_{121}=-1, & \gamma_{122}=0, \\
\gamma_{211}=0, & \gamma_{212}=1, & \gamma_{221}=0, & \gamma_{222}=-1 .
\end{array}\right. \\
& \Delta_{a} \equiv\left|\begin{array}{cc}
a_{1} & a_{2} \\
-a_{1} & -a_{2}
\end{array}\right|=0, \quad \Delta_{a}^{\prime} \equiv\left|\begin{array}{cc}
a_{1}-a_{2} & 0 \\
0 & a_{1}-a_{2}
\end{array}\right|=\left(a_{1}-a_{2}\right)^{2} \text {. }
\end{aligned}
$$

We may verify directly that conditions (5) are satisfied; or we may verify relations (4), employing relations (1) which bere become

$$
e_{1} e_{1}=e_{1}, \quad e_{1} e_{2}=-e_{1}, \quad e_{2} e_{1}=e_{2}, \quad e_{2} e_{2}=-e_{2} .
$$

$S_{7} . \quad\left\{\begin{array}{llll}\gamma_{111}=1, & \gamma_{112}=0, & \gamma_{121}=0, & \gamma_{122}=1, \\ \gamma_{211}=-1, & \gamma_{212}=0, & \gamma_{221}=0, & \gamma_{222}=-1\end{array}\right.$

* When $F$ does not have modulus 2, we may take for $S_{5}$ the set

$\gamma_{111}=1, \quad \gamma_{112}=0, \quad \gamma_{121}=0, \quad \gamma_{122}=-1, \quad \gamma_{211}=0, \quad \lambda_{212}=-1, \quad \gamma_{221}=-1, \quad \gamma_{232}=0$. Then $\Delta_{a}=\Delta_{a}^{\prime}=-\left(a_{1}^{2}+a_{2}^{2}\right)$; while (5) faile for $i=2, k=2, l=1, t=1$, since $-1 \neq+1$. 


$$
\Delta_{a} \equiv\left|\begin{array}{cc}
a_{1}-a_{2} & 0 \\
0 & a_{1}-a_{2}
\end{array}\right|=\left(a_{1}-a_{2}\right)^{2}, \quad \Delta_{a}^{\prime} \equiv\left|\begin{array}{rr}
a_{1} & a_{2} \\
-a_{1} & -a_{2}
\end{array}\right|=0 .
$$

That conditions (5) are satisfied follows from the fact that the set $S_{7}$ can be derived from the set $S_{6}$ by interchanging $\gamma_{i k s}$ with $\gamma_{k i s}$.

\section{Second Definition of a System of Complex Numbers.}

We consider a system of elements $A=\left(a_{1}, a_{2}, \ldots, a_{n}\right)$ each uniquely defined by $n$ marks of the field $F$ together with their sequence. The marks $a_{1}, \ldots a_{n}$ are called the coördinates of $A$. The element $(0,0, \ldots, 0)$ is called zero and designated 0 .

Addition of elements is defined thus :

$$
A+B=\left(a_{1}+b_{1}, a_{2}+b_{2}, \cdots, a_{n}+b_{n}\right) .
$$

It follows that there is an element $D=\left(a_{1}-b_{1}, \ldots, a_{n}-b_{n}\right)$ such that $D+B=A$.

Consider a second rule of combination of the elements having the properties : *

1. For any two elements $A$ and $B$ of the system, $A \cdot B$ is an element of the system whose coördinates are bilinear functions of the coördinates of $A$ and $B$, with fixed coefficients belonging to $F$.

2. $(A \cdot B) \cdot C=A \cdot(B \cdot C)$, if $A \cdot B, B \cdot C,(A \cdot B) \cdot C, A \cdot(B \cdot C)$ belong to the system.

3. There exists in the system an element $I$ such that $A \cdot I=A$ for every element $A$ of the system.

4. There exists in the system at least one element $A$ such that $A \cdot Z \neq 0$ for any element $Z \neq 0$.

That any sysstem of elements given by the second definition is a system of complex numbers according to the usual (first) definition is next shown. $\dagger$ From 1. and (8) follows the distributive law :

$$
A \cdot(B+C)=(A \cdot B)+(A \cdot C) .
$$

For any element $I$ satisfying $3, I \cdot B=B$ for every element $B$.

In proof, let $A$ be one of the elements satisfying 4 , and let $B^{\prime}$ be such that $B+B^{\prime}=0$ (see above). Then by 2

* Note that 3 assumes the existence of a right-hand identity element. Postulate 4 is milder than the assumption of a left-hand identity $I^{\prime}$, while from the existence of $I^{\prime}$ would follow 4 , $A$ being taken as $I^{\prime}$.

† The inverse is true. That a system of complex numbers contains an identity element (so that 3 and 4 follow) is shown in LIE-ScheFFers, p. 614. 


$$
A \cdot(I \cdot B)=(A \cdot I) \cdot B=A \cdot B
$$

Hence, by (9) applied twice,

$$
A \cdot\left[(I \cdot B)+B^{\prime}\right]=(A \cdot B)+\left(A \cdot B^{\prime}\right)=A \cdot\left(B+B^{\prime}\right)=A \cdot 0 .
$$

By $1, A \cdot 0=0$. Hence by $4,(I \cdot B)+B^{\prime}=0$, so that $I \cdot B=B$.

There is an unique element $I$ satisfying 3.

For, let $I$ be the given element and $I^{\prime}$ a second element, each satisfying 3 . Then $I^{\prime} \cdot I=I^{\prime}$ by 3 . By the preceding theorem, $I^{\prime} \cdot B=B$ for every $B$, whence $I^{\prime} \cdot I=I$. It follows that $I^{\prime}=I$.

There is an unique element $I$ such that $I \cdot B=B$ for every $B$.

For, let $I_{1}$ be one such element and let $I$ be the unique element satisfying 3 . Then $I_{1} \cdot B=B$ gives $I_{1} \cdot I=I$, while 3 gives $I_{1} \cdot I=I_{1}$. Hence $I_{1}=I$.

From the three preceding results it follows that there is an unique element $I$ such that $A \cdot I=I \cdot A=A$ for every $A$.

To pass to the form of representation used in the first.definition, we make $A \equiv\left(a_{1}, a_{2}, \cdots, a_{n}\right)$ correspond to $a \equiv a_{1} e_{1}+a_{2} e_{2}+\cdots+a_{n} e_{n}$. In view of 1 , there exist constant marks $\gamma_{i k s}$ of $F$ such that relation (3) holds and, as special cases, relations (1). Condition 2 thus leads to relations (5). Since there is an unique solution * $X=I$ of $A \cdot X=A$, where $A$ satisfies 4 , the determinant $\Delta_{a}$ does not vanish for every $a_{1}, \ldots, a_{n}$. Since there is an unique solution $X=I$ of $X \cdot A=A$, where $A$ is such that $Z A \neq 0$ if $Z \neq 0$, $\Delta_{a}^{\prime}$ does not vanish for every $a_{1}, \cdots, a_{n}$. Since conditions (5), (6), and (7) are satisfied, the system of elements forms a system of complex numbers.

\section{Independence of the Postulates 1, 2, 3, 4 .}

For $i=1,2,3,4$, we exhibit a system $\Sigma_{i}$ of elements for which the $i$ th postulate fails, while the remaining three postulates hold.

$\Sigma_{1}$. Take $A \cdot B=A$. Or take $A \cdot B=A+B$ with $I=0$ and $A=0$ in 4 .

$\Sigma_{2}$. Take $n=2$, and for $A \cdot B$ take the law of combination

$$
\left(a_{1}, a_{2}\right)\left(\alpha_{1}, \alpha_{2}\right)=\left(a_{1} \alpha_{1}+a_{1} \alpha_{2}+a_{2} \alpha_{2}, a_{2} \alpha_{1}\right) .
$$

Then 3 is satisfied for $I=(1,0)$, and 4 for $A=(0,1)$ since

$$
(0,1)\left(z_{1}, z_{2}\right)=\left(z_{2}, z_{1}\right) \text {. }
$$

But 2 fails for $A=(0,1), B=(1,0), C=(0,1)$.

$\Sigma_{3}$. We employ the system $S_{7}$. Hence $A \cdot B$ is given by

* For, by the proof of the first theorem, $X \cdot B=B$ for every $B$, whence $X=I$. 


$$
\left(a_{1}, a_{2}\right)\left(b_{1}, b_{2}\right)=\left(a_{1} b_{1}-a_{2} b_{1}, a_{1} b_{2}-a_{2} b_{2}\right) .
$$

Hence 1 and 2 hold. Since $(1,0)\left(z_{1}, z_{2}\right)=\left(z_{1}, z_{2}\right), 4$ holds. To show that 3 fails, we note that $A \cdot B=A$ requires

$$
b_{1}\left(a_{1}-a_{2}\right)=a_{1}, \quad b_{2}\left(a_{1}-a_{2}\right)=a_{2},
$$

so that $b_{1}$ and $b_{2}$ are not independent of $a_{1}$ and $a_{2}$.

$\Sigma_{4}$. We employ the system $S_{6}$. Hence $A \cdot B$ is given by

$$
\left(a_{1}, a_{2}\right)\left(b_{1}, b_{2}\right)=\left(a_{1} b_{1}-a_{1} b_{2}, a_{2} b_{1}-a_{2} b_{2}\right) \text {. }
$$

Hence 1 and 2 hold. Also 3 holds for $I=(1,0)$. But 4 fails since

$$
\left(a_{1}, a_{2}\right)(1,1)=(0,0) .
$$

The University of Chicago, August, 1902. 